\title{
Phases of the excitonic condensate in two-layer graphene
}

\author{
Yevhen F. Suprunenko, Vadim Cheianov, and Vladimir I. Fal'ko \\ Department of Physics, Lancaster University, Lancaster LAl 4YB, United Kingdom
}

(Received 23 June 2012; published 4 October 2012)

\begin{abstract}
Two graphene monolayers that are oppositely charged and placed close to each other are considered. Taking into account valley and spin degeneracy of electrons, we analyze the symmetry of the excitonic insulator states in such a system and build a phase diagram that takes into account the effect of the symmetry breaking due to the external in-plane magnetic field and the carrier density imbalance between the layers.
\end{abstract}

DOI: 10.1103/PhysRevB.86.155405

PACS number(s): 73.63.-b, 73.22.Gk, 73.21.-b

\section{INTRODUCTION}

The excitonic insulator ${ }^{1-5}$ was predicted theoretically four decades ago in 3D semiconductors and then in spatially separated layers of electrons and holes. ${ }^{6,7}$ Since then, an excitonic insulator has been searched for in a variety of systems. The excitonic insulator is a material where the electron-hole excitonic correlations lead to the formation of a gapped state characterized by the order parameter resembling a superfluid condensate of excitons. Such a correlated state has been observed in double-quantum well semiconductor structures in quantizing magnetic fields. ${ }^{8-14}$ After the experimental discovery of graphene ${ }^{15-18}$ it has been discussed as a possible candidate for experimental realization of the excitonic insulator state ${ }^{19-24}$ sparking the on-going debate ${ }^{25-27}$ about the critical temperature $T_{c}$ of the excitonic condensate transition in a two-layer graphene system. Various estimations of $T_{c}$ for such a system lay in a wide region of magnitudes from milli-Kelvins ${ }^{25,27}$ up to Kelvins ${ }^{28-31}$ and further up to the room temperature..$^{22,23}$ The considered system ${ }^{20-23,25-27}$ consists of two parallel, separately controlled graphene monolayers, in which external gates induce a finite density of electrons in the layer 1 and holes in the layer 2 [see Fig. 1(a)]. Recently, the two-layer graphene system has been obtained experimentally. ${ }^{32-37}$

In this paper, we extend the existing theory of the excitonic insulator state in a two-layer graphene system: we analyze a symmetry of the excitonic insulator and classify its phases. As a result, a phase diagram of the excitonic insulator is built that takes into account the effect of the symmetry breaking due to the Zeeman splitting and the asymmetry between electron/hole densities in the layer 1 and 2. A phase diagram, Fig. 1(b), contains three phases: $B, B^{\prime}$, and $A_{1}$. Transitions between phases are found to be of the first order. These transitions are subject to the use of an in-plane magnetic field and a variation of external gate voltages, leading to different charge carriers densities in layers: the density of all electrons $n_{1 e}$ in layer 1 (which corresponds to the Fermi energy $E_{F}^{(1)}=\hbar v \sqrt{\pi n_{1 e}} / 2$ ), and density of holes $n_{2 h}$ in layer 2 (which corresponds to the negative Fermi energy in the layer $2, E_{F}^{(2)}=-\hbar v \sqrt{\pi n_{2 h}} / 2$ ).

The $B$ phase, Fig. 1(b), exists when there is no magnetic field and charge carriers densities are the same in both layers $n_{1 e}=n_{2 h}$ (i.e., when $E_{F}^{(1)}=\left|E_{F}^{(2)}\right|$ ). The $B^{\prime}$ phase exists at the same condition $n_{1 e}=n_{2 h}$ but when an in-plane magnetic field is applied, which causes a Zeeman splitting of energies of electrons with different spin projections. The $A_{1}$ phase exists when a symmetry of charge carriers density is violated, e.g., $n_{1 e}>n_{2 h}$, and when the corresponding splitting of Fermi energies $E_{F}^{(1)}-\left|E_{F}^{(2)}\right|$ is equal to the Zeeman splitting due to an in-plane magnetic field.

The diversity of obtained phases is due to the high symmetry of the normal ground state, which can be broken in several different ways leading to a variety of phases possessing different symmetry groups. A well-known example of the system with the diversity of phases due to various normal state symmetry breaking is liquid Helium-3. ${ }^{38-42}$ In liquid Helium-3, the symmetry of the order parameter can be changed by correspondent external parameters, leading to phase transitions.

The analysis in this paper is organised as follows. Section II describes the theoretical model of the considered system. Pairing of electrons and holes within mean-field theory is introduced in the Sec. III. Section IV provides symmetry analysis and the phase classification of the excitonic correlated state. Section V contains detailed description of the most symmetric phases, their properties are summarizes in Table I. Results are discussed in Sec. VI.

\section{TWO-LAYER HAMILTONIAN}

Graphene ${ }^{43-45}$ is a gapless semiconductor with the Fermi surface consisting of two distinct points, $\mathbf{K}_{+}$and $\mathbf{K}_{-}$, called valleys. Near these Fermi points, electrons have a linear dispersion $E(p)= \pm v p$, with a velocity $v \approx 10^{8} \mathrm{~cm} / \mathrm{sec},{ }^{16}$ here $p=|\mathbf{p}|, \mathbf{p}=\mathbf{k}-\mathbf{K}_{ \pm}$is the momentum of an electron relative to the Fermi point. Using external gates, one can independently tune the carrier density in each of the two graphene flakes. ${ }^{16}$ Neglecting tunneling, the electrons in the two layer graphene system initially can be described with the Hamiltonian $\hat{H}_{2 \text { layer }}=\hat{H}_{\text {s.p. }}+\hat{H}_{11}+\hat{H}_{22}+\hat{H}_{12}$, here the single-particle part of the Hamiltonian is

$$
\hat{H}_{\text {s.p. }}=\sum_{l, \zeta, \mathbf{p}, s}\left(s v p-E_{F}^{(l)}\right) a_{l, \zeta, \mathbf{p}, s}^{\dagger} a_{l, \zeta, \mathbf{p}, s},
$$

the operators $a_{l, \zeta, \mathbf{p}, s}^{\dagger}\left(a_{l, \zeta, \mathbf{p}, s}\right)$ create (annihilate) an electron on the $l=1,2$ layer on the $s=+/-$ conduction or valence band with momentum $\mathbf{p}=p\left(\cos \phi_{\mathbf{p}}, \sin \phi_{\mathbf{p}}\right), E_{F}^{(1)}$ and $E_{F}^{(2)}$ are the Fermi energies, which correspond to charge carrier densities in the layers. The index $\zeta$ denotes four different pairs of spin projection $(\uparrow, \downarrow)$ and valleys $\left(\mathbf{K}_{+}, \mathbf{K}_{-}\right)$. In the Hamiltonian $\hat{H}_{2 \text { layer }}$, the terms $\hat{H}_{11}$ and $\hat{H}_{22}$ take into account the intralayer interaction. These terms can be ignored in the following studies, provided that one uses a screened inter-layer 
(a)

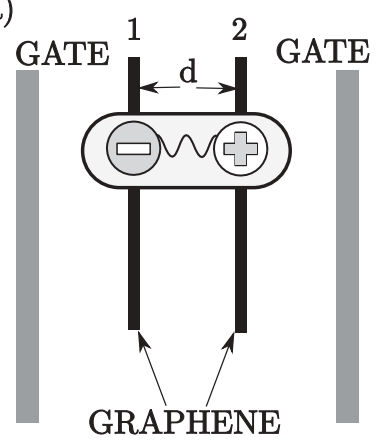

(b)

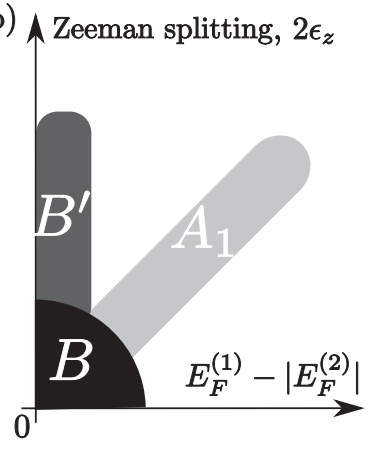

FIG. 1. (a) The excitonic condensation due to an electron-hole pairing is studied in the system of two spatially separated graphene monolayers with an excess of electrons on layer 1 and a lack of electrons on layer 2. (b) The schematic phase diagram of the excitonic condensation in the system at different values of a Zeeman splitting and different values of the asymmetry between Fermi energies in layer 1 and $2, \epsilon_{Z}=\mu_{B}|\mathbf{h}|$ is the Zeeman energy in an in-plane magnetic field $\mathbf{h}$.

interaction in the term $\hat{H}_{12}$. Hence in $\hat{H}_{12}$, we keep only those terms that contribute to the BCS mean-field theory, ${ }^{46}$ absorbing other contributions into a renormalization of the velocity and the Fermi energy in the single particle part (1) of the Hamiltonian

$$
\begin{aligned}
\hat{H}_{12}= & -\sum_{\mathbf{p}, \mathbf{p}^{\prime}, s, s^{\prime}} V\left(\left|\mathbf{p}-\mathbf{p}^{\prime}\right|\right) \frac{1+s s^{\prime} \cos \left(\phi_{\mathbf{p}}-\phi_{\mathbf{p}^{\prime}}\right)}{2} \\
& \times \sum_{\zeta, \zeta^{\prime}} a_{1, \zeta, \mathbf{p}, s}^{\dagger} a_{2, \zeta^{\prime}, \mathbf{p}^{\prime},-s^{\prime}}^{\dagger} a_{1, \zeta, \mathbf{p}^{\prime}, s^{\prime}} a_{2, \zeta^{\prime}, \mathbf{p},-s} .
\end{aligned}
$$

The scattering process, described by $\hat{H}_{12}$, is shown on Fig. 2. The function $V(q)$ denotes a screened Coulomb interaction in the static limit $V(q)=V(q, \omega \ll q)$. The factor $\left[1+s s^{\prime} \cos \left(\phi_{\mathbf{p}}-\phi_{\mathbf{p}^{\prime}}\right)\right] / 2$ in Eq. (2) reflects chiral properties of electrons related to the sublattice composition of electronic Bloch wave functions. ${ }^{44,45}$ These chiral properties of electrons result in the suppressed backwards scattering if an electron does not change the energy band upon scattering $\left(s s^{\prime}=+\right)$, otherwise $\left(s s^{\prime}=-\right)$ the electron can not forward scatter. ${ }^{47}$

\section{EXCITONIC PAIRING, MEAN-FIELD ORDER PARAMETER}

The excitonic insulator state of the electron-hole liquid is characterized by the electron-hole correlations on the Fermi surface, Fig. 3. Mathematically, it means that in the excitonic

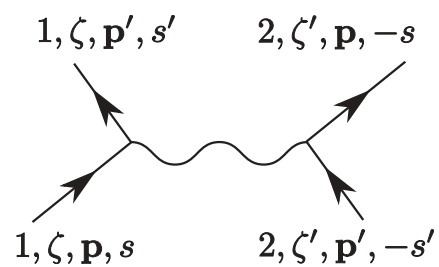

FIG. 2. A typical transition which is described by $\hat{H}_{12}$ in Eq. (2). Indices $l=1$ or $2, \zeta, \mathbf{p}$, and $s$ denote a layer, a pair of the spin projection and valley, a momentum of an electron, and a conduction $s=+$ or valence $s=-$ band

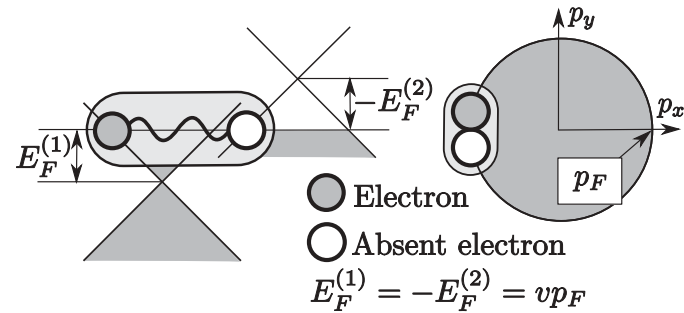

FIG. 3. The excitonic electron-hole bound state in the two-layer graphene. The left-hand side of the figure shows the electron's spectrum in graphene layers 1 and 2. An electron on the Fermi surface in the layer 1 is shown as a fulfilled circle. Absence of an electron on the Fermi surface in layer 2 is shown as an empty circle. Closed line around both circles represents an excitonic pairing, which is developed due to a Coulomb interaction (shown as a wavy line). The right-hand side of the figure shows the coincided Fermi circles in both layers at Fermi momentum $p_{F}$.

insulating state there is a nonvanishing ground-state average $\mathrm{F}$ of electron operators:

$$
\mathrm{F}_{\zeta \zeta^{\prime}, s}(\mathbf{p})=\left\langle a_{2, \zeta^{\prime}, \mathbf{p},-s}^{\dagger} a_{1, \zeta, \mathbf{p},+s}\right\rangle .
$$

For the existence of the nonzero ground-state average $\mathrm{F}$, it is crucial that Fermi surfaces for electrons and holes coincide. ${ }^{46,48}$ Due to the electron-hole symmetry of the energy spectrum in graphene, the electron-hole excitonic correlations (3) in the considered system are most developed when the density of electrons in the layer 1 is equal to the density of holes in the layer $2, n_{1 e}=n_{2 h}$, or, in terms of Fermi energies $E_{F}^{(1)}=-E_{F}^{(2)}$, Fig. 3. However, apart from this condition there can be certain other external conditions when excitonic correlations (3) can be developed. Thus, although the excitonic insulator state disappears when the symmetry $n_{1 e}=n_{2 h}$ is violated by external gates, we show below that excitonic correlations can be restored by the in-plane magnetic field. Based on the detailed analysis of excitonic correlations in monolayer graphene in the in-plane magnetic field, which is done by Aleiner and co-authors, ${ }^{19}$ we show that the excitonic insulator state can exist in various phases in the two-layer graphene system.

In order to study phases of the excitonic insulator state at different external conditions, firstly, we apply the standard mean-field approximation. ${ }^{46} \mathrm{We}$ assume that the product of the operators $a_{2, \zeta^{\prime}, \mathbf{p},-s}^{\dagger} a_{1, \zeta, \mathbf{p}, s}$ weakly deviates from its nonvanishing ground-state average. We expand the interacting part $\hat{H}_{12}$, Eq. (2), of the Hamiltonian $\hat{H}_{2 \text { layer }}$ up to the linear order with respect to these small deviations and neglect constant terms. The mean-field Hamiltonian of the system becomes

$$
\hat{H}_{\mathrm{mf}}=\hat{H}_{\text {s.p. }}+\sum_{\mathbf{p}, s, \zeta, \zeta^{\prime}}\left[a_{1, \zeta, \mathbf{p}, s}^{\dagger} \Delta_{\zeta \zeta^{\prime}, s}(\mathbf{p}) a_{2, \zeta^{\prime}, \mathbf{p},-s}+\text { H.c. }\right],
$$

where H.c. stands for "Hermitian conjugate," and

$$
\begin{aligned}
\Delta_{\zeta \zeta^{\prime}, s}(\mathbf{p})= & -\sum_{\mathbf{p}^{\prime}, s^{\prime}} \mathrm{F}_{\zeta \zeta^{\prime}, s^{\prime}}\left(\mathbf{p}^{\prime}\right) V\left(\left|\mathbf{p}-\mathbf{p}^{\prime}\right|\right) \\
& \times \frac{1+s s^{\prime} \cos \left(\phi_{\mathbf{p}}-\phi_{\mathbf{p}^{\prime}}\right)}{2} .
\end{aligned}
$$


Quantities $\Delta_{\zeta \zeta^{\prime}, s}(\mathbf{p})$ form the matrix $\Delta$ of the order parameter. Index $\zeta$ denotes four different pairs of spin projections and valleys ( $\uparrow \mathbf{K}_{+}, \uparrow \mathbf{K}_{-}, \downarrow \mathbf{K}_{+}, \downarrow \mathbf{K}_{-}$). Thus in the spin $\otimes$ valley space the order parameter is given by the $4 \times 4$ matrix $\Delta$ with matrix elements given by Eq. (5). For brevity, we omit index $s$ and momentum $\mathbf{p}$ in the notation for the order parameter $\Delta$.

For further analysis it is convenient to rewrite the Hamiltonian (4) as follows: $\hat{H}_{\mathrm{mf}}=\sum_{\zeta, \mathbf{p}, \mathbf{s}} \Psi_{\zeta, \mathbf{p}, \mathbf{s}}^{\dagger} H_{\mathrm{mf}}(\mathbf{p}, s) \Psi_{\zeta, \mathbf{p}, \mathbf{s}}$, where $\Psi_{\zeta, \mathbf{p}, \mathbf{s}}=\left(a_{1, \zeta, \mathbf{p},+\mathbf{s}}, a_{2, \zeta, \mathbf{p},-\mathbf{s}}\right)^{T}$ and

$$
H_{\mathrm{mf}}(\mathbf{p}, s)=\left(\begin{array}{cc}
\left(s v p-E_{F}\right) \mathbb{1} & \Delta \\
\Delta^{\dagger} & -\left(s v p-E_{F}\right) \mathbb{1}
\end{array}\right) .
$$

Here, all elements of the matrix $H_{\mathrm{mf}}$ are $4 \times 4$ matrices in the spin $\otimes$ valley space: diagonal elements have structure of the identity matrix $\mathbb{1}$ in this space, whereas $\Delta$ is given by some $4 \times 4$ matrix, whose structure is identified in this paper for each phase of the excitonic correlated state. The matrix of the order parameter $\Delta$ describes the correlations between conduction/valence electrons in the layers 1 and 2 below a critical temperature $T_{c}$.

Nevertheless, the phase classification can be made regardless of the value of the transition temperature $T_{c}$. Assuming that the excitonic insulator state can be observed in two-layer graphene system, we analyze the symmetry of the mean field Hamiltonian (4) and the order parameter $\Delta$. As a result the classification of all phases of the excitonic insulating state of the two-layer graphene system is presented in the next section, and a detailed discussion of each phase is presented in Sec. V.

\section{SYMMETRY ANALYSIS OF THE CORRELATED STATE}

The analysis in this section is based on the idea of breaking of the initial symmetry of the hamiltonian by the order parameter. The initial symmetry group $G$ of the Hamiltonian $\hat{H}_{2 l a y e r}$ is formed by global unitary transformations of an electronic single-particle state in the four-component spin $\otimes$ valley space independently in the layers 1 and 2 . These transformations are represented by independent matrices $\mathrm{U}^{(1)}$ and $\mathrm{U}^{(2)}$ in layers 1 and 2, respectively. Therefore the group $G$ is given by the direct product of corresponding unitary groups $U_{4}$ :

$$
G=U_{4}^{(1)} \times U_{4}^{(2)} .
$$

Unitary group $U_{4}^{(l)}, l=1,2$, consists of $4 \times 4$ unitary matrices $\mathrm{U}^{(l)}$, which perform transformations of electron's operators in the $l$ th layer as follows:

$$
a_{l, \zeta, \mathbf{p}, s} \rightarrow \sum_{\zeta^{\prime}} \mathrm{U}_{\zeta \zeta^{\prime}}^{(l)} a_{l, \zeta^{\prime}, \mathbf{p}, s}
$$

Thus, as it is seen from Eqs. (4) and (6), under symmetry transformations (8) the order parameter $\Delta$ transforms as

$$
\Delta \longrightarrow \mathrm{U}^{(1) \dagger} \Delta \mathrm{U}^{(2)} \text {. }
$$

This implies that the Hamiltonian of the system is not invariant under the action of the group $G$ any longer. However, for any fixed nonzero $\Delta$, there is always some subgroup $H$ of the group $G, H \subset G, H \neq G$, such that all transformations from the group $H$ do not transform $\Delta$, i.e., the order parameter $\Delta$ remains invariant:

$$
\mathrm{U}_{H}^{(1) \dagger} \Delta \mathrm{U}_{H}^{(2)}=\Delta .
$$

Such transformations $\mathrm{U}_{H}^{(1)}$ in layer 1 and $\mathrm{U}_{H}^{(2)}$ in layer 2 form a symmetry group $H$ :

$$
\left(\begin{array}{cc}
\mathrm{U}_{\mathrm{H}}^{(1)} & 0 \\
0 & \mathrm{U}_{H}^{(2)}
\end{array}\right) \in H \subset G .
$$

Only transformations from the group $H$ leave the ground state of the excitonic insulator invariant, i.e., only these transformations leave the mean-field Hamiltonians (4) and (6) invariant:

$$
\left(\begin{array}{cc}
\mathrm{U}_{\mathrm{H}}^{(1) \dagger} & 0 \\
0 & \mathrm{U}_{H}^{(2) \dagger}
\end{array}\right) H_{\mathrm{mf}}(\mathbf{p}, s)\left(\begin{array}{cc}
\mathrm{U}_{\mathrm{H}}^{(1)} & 0 \\
0 & \mathrm{U}_{H}^{(2)}
\end{array}\right)=H_{\mathrm{mf}}(\mathbf{p}, s) .
$$

Thus the symmetry group $G$ of the initial uncorrelated normal ground state of the system is broken down to the symmetry group $H$ of the ground state of the excitonic insulator.

All transformations from $G$, which are not included in $H$, form the factor space $G / H$. These transformations change the order parameter $\Delta$, however, they do not change the energy of the corresponding ground state. Therefore the manifold of all matrices $\Delta$, which can be obtained by transformations from $G / H$, form a degeneracy space of the order parameter. Consequently, the manifold of the correspondent ground states form a phase of the correlated state. It is important to notice, that all these ground states within the same phase are described by the same symmetry group $H$, which is a symmetry group of the order parameter. Therefore phases of a correlated state can be classified by the symmetry group $H$ and the degeneracy space of the order parameter.

The phase classification presented in this paper is also reminiscent of the classification of the various degeneracy spaces of the order parameter in liquid Helium-3. ${ }^{38-40,42}$ This classification principle was used in the determination of superconducting phases in nontrivial superconductors ${ }^{46}$ and superfluid phases in liquid Helium-3. ${ }^{41,49}$

In order to classify phases of the excitonic insulating state, we are going to classify the degeneracy spaces of order parameters with the same symmetry. For this, we consider the condition (10) and use the method of a singular value decomposition. ${ }^{50}$ It allows us to represent an arbitrary matrix $\Delta$ as a product of a unitary matrix $\widetilde{\mathrm{V}}^{\dagger}$, a diagonal matrix $\mathrm{D}$ with real nonnegative numbers on the diagonal, and another unitary matrix V. Applying the singular value decomposition to the order parameter at any given values of $s$ and $\mathbf{p}$ we obtain

$$
\Delta=\widetilde{\mathrm{V}}_{s}^{\dagger}(\mathbf{p}) \mathrm{D}_{s}(\mathbf{p}) \mathrm{V}_{s}(\mathbf{p}) .
$$

However, first of all, we notice that in the considered system, the lowest ground-state energy is realized when matrices $\mathrm{V}, \widetilde{\mathrm{V}}$ do not depend on momentum $\mathbf{p}$ and index $s= \pm$. The reason for this is that in such a case in the expression for the ground-state energy there is a cancellation of the product of matrices $\mathrm{V}$ and $\mathrm{V}^{\dagger}\left(\widetilde{\mathrm{V}}\right.$ and $\left.\widetilde{\mathrm{V}}^{\dagger}\right)$ into a unit matrix. Such a cancellation leads to the maximal negative contribution to the ground-state energy, and therefore the energy of the ground state achieves its minimal value. If we assume that unitary matrices in Eq. (13) depend on momentum $\mathbf{p}$ and index $s= \pm$, then unitary matrices at different momenta $\mathbf{p}, \mathbf{p}^{\prime}$ and 
different indices $s, s^{\prime}$ do not cancel each other, which increases the ground-state energy comparatively to the previous case. Thus we conclude that in order to realize the lowest energy of the ground state, matrices $\widetilde{V}$ and $\mathrm{V}$ can not depend on the momentum $\mathbf{p}$ and index $s$. Therefore the singular value decomposition of the matrix of order parameter becomes

$$
\Delta=\widetilde{\mathrm{V}}^{\dagger} \mathrm{DV},
$$

where in the right-hand side of the equation (14) only matrix $\mathrm{D}$ depends on $\mathbf{p}$ and $s$, but for brevity we omit these indices.

Second, all transformations from the group $G$, including those from the factor space $G / H$, do not change the diagonal elements of the matrix $\mathrm{D}$, but change matrices $\widetilde{\mathrm{V}}, \mathrm{V}$ into any other unitary matrices. Thus if we introduce the notations

$$
\tilde{\mathrm{V}}^{\prime \dagger} \equiv \mathrm{U}^{(1) \dagger} \tilde{\mathrm{V}}^{\dagger}, \quad \mathrm{V}^{\prime} \equiv \mathrm{V} \mathrm{U}^{(2)},
$$

then under the transformation (8) and (9), the order parameter will transform in the following way:

$$
\Delta=\tilde{\mathrm{V}}^{\dagger} \mathrm{DV} \longrightarrow \Delta^{\prime}=\tilde{\mathrm{V}}^{\prime \dagger} \mathrm{DV}^{\prime} .
$$

Thus under this transformation the diagonal matrix D does not change. Recall that the degeneracy space of the order parameter is obtained by acting on the order parameter $\Delta$ by all transformations from the group $G$ (here transformations from subgroup $H$ will not change the order parameter while remaining transformations from factor-space $G / H$ will create the degeneracy space of the order parameter). As long as only matrices $\mathrm{V}$ and $\widetilde{\mathrm{V}}$ are changed by transformations from $G$, we obtain that the degeneracy space of the order parameter and a phase of the correlated state are determined only by the diagonal elements of the matrix D.

Finally, from the condition (10) we have found that all possible degeneracy spaces of the order parameter are classified by numbers of equal and different diagonal elements in matrix D. In the case of physically relevant phases, there are additional restrictions on the diagonal elements of matrix D. Thus among all possible matrices $\Delta$ only physically relevant order parameters satisfy the self-consistency equation. For the phase classification, it is sufficient to consider the BCS self-consistency equation for the order parameter. Diagonalizing the self-consistency equation by unitary matrices from Eq. (14), one obtains four equations for diagonal elements of the matrix D, each equation corresponds to some value of index $\zeta$. These equations have the same structure and depend on the Fermi momentum $p_{F}$. If the Fermi momentum $p_{F}$ is the same for all types of electrons (for all indices $\zeta$ ), then these four self-consistency equations are identical, and apart from a trivial zero solution they have the same nonzero solution. Hence in such situation in physically relevant phases, the arbitrary diagonal element in matrix $\mathrm{D}$ can be equal either to other nonzero diagonal elements, or be equal to a zero. The application of the in-plane magnetic field, in principle, changes such a description because of Zeeman splitting. However, in the case where the Fermi energy is much greater than the Zeeman energy, $E_{F} \gg \epsilon_{Z}$, the magnetic field does not change the situation essentially as long as it is possible to neglect the difference between Fermi momenta for electrons with opposite spin projections in the self-consistency equations. Therefore four self-consistency equations on four diagonal elements of the matrix D become approximately identical also when relatively small in-plane magnetic field $\left(\epsilon_{Z} \ll E_{F}\right)$ is applied.

The nonzero solution of these equations is given by the gap function $g_{s}(\mathbf{p})$, which at the Fermi surface $\left(s=+,|\mathbf{p}|=p_{F}\right)$ determines a gap in the single-particle excitation spectrum. Thus we conclude that in all physically relevant phases the matrix D in the singular value decomposition (14) of the order parameter $\Delta$ consists of zeros or non-negative diagonal elements, which approximately are equal to the gap function $g_{s}(\mathbf{p})$.

Substituting the obtained result into Eq. (14), we extract the gap function as a multiplier. Thus we conclude that the order parameter $\Delta$ in all physically relevant phases has a form

$$
\Delta \cong g_{s}(\mathbf{p}) \tilde{\mathrm{V}}^{\dagger} \mathrm{DV} .
$$

Here, the matrix $D$ is a diagonal matrix with 0 or 1 on the diagonal. The representation (17) becomes approximate in the case of the applied in-plane magnetic field with the condition $\epsilon_{Z} \ll E_{F}$. The dependence of the order parameter $\Delta$ on variables $s$ and $\mathbf{p}$ is completely given by the function $g_{s}(\mathbf{p})$.

Having the matrix of the order parameter provided, the symmetry group $H$ is found from Eq. (10). For this, the matrix of the order parameter is represented as a single-value decomposition (14). The constant matrices $\mathrm{V}$ and $\widetilde{\mathrm{V}}$ are absorbed into matrices $\mathrm{U}_{H}^{(1)}$ and $\mathrm{U}_{H}^{(2)}$ of the global symmetry transformations from the symmetry group $H$. Then Eq. (10) connects two unitary matrices $\mathrm{VU}_{H}^{(1)} \mathrm{V}^{\dagger}$ and $\tilde{\mathrm{V}} \mathrm{U}_{H}^{(2)} \widetilde{\mathrm{V}}^{\dagger}$ and the diagonal matrix $\mathrm{D}$ with 0 or 1 on the diagonal. Thus the matrices $\mathrm{U}_{H}^{(1)}$ and $\mathrm{U}_{H}^{(2)}$ of transformations from the symmetry group $H$ are obtained.

\section{PHASES}

In this section, we provide a detailed description of phases of excitonic insulator state in two-layer graphene system. Results of this section are summarized in Table I.

\section{A. The $B$ phase}

Firstly, we consider the situation when there is no external magnetic field and when the charge carrier densities in layers are the same $n_{1 e}=n_{2 h}$. In such case, the symmetry group $G$ of the two-layer Hamiltonian of the system in normal state is given in Eq. (7). Under the mentioned conditions, the Fermi circle in the conduction band in layer 1 coincides with the Fermi circle in the valence band in layer 2 due to the electron-hole symmetry in graphene. Hence the nonvanishing ground-state average F, Eq. (3), can be formed by all species of electrons. Taking into account that the ground state with the lower energy is more stable, we consider the phase when excitonic correlations are developed among all species of electrons. In such cases, the order parameter matrix $\Delta$ and the matrix D, Eq. (17), are not degenerate matrices. Moreover, because there is only one Fermi circle for all species of electrons, the most stable ground state is characterized by the matrix D in Eq. (17) with equal nonzero diagonal elements, i.e., $\mathrm{D}$ is an identity matrix. As discussed in the previous section, this conclusion follows from the consideration of the self-consistency equation on the order parameter. Thus 
TABLE I. The phase classification of the excitonic insulating state with respect to following two external parameters: (1) Zeeman energy $\epsilon_{Z}=\mu_{B}|\mathbf{h}|$ in an in-plane magnetic field $\mathbf{h}$ and (2) an asymmetry between the electron density $n_{1 e}$ in the layer 1 and the hole density $n_{2 h}$ in the layer 2 .

\begin{tabular}{|c|c|c|c|c|c|c|}
\hline $\begin{array}{l}\text { External } \\
\text { conditions }\end{array}$ & $\begin{array}{c}\text { Symmetry group } G \\
\text { of the two-layer } \\
\text { Hamiltonian }\end{array}$ & Phase & $\begin{array}{c}\text { Matrix structure } \\
\text { of the order } \\
\text { parameter } \Delta \\
\text { (here } \mathrm{V}, \widetilde{\mathrm{V}} \in U_{4} \text { ) }\end{array}$ & $\begin{array}{c}\text { Symmetry group } H \\
\text { of the order } \\
\text { parameter } \Delta\end{array}$ & $\operatorname{dim}[G / H]$ & $\begin{array}{c}\text { Single } \\
\text { particle } \\
\text { spectrum }\end{array}$ \\
\hline \multirow{4}{*}{$\begin{array}{l}n_{1 e}=n_{2 h} \\
\epsilon_{Z}=0\end{array}$} & \multirow{4}{*}{$U_{4}^{(1)} \times U_{4}^{(2)}$} & $B$ & $\mathrm{~V}$ & $U_{4}^{(1,2)}$ & 16 & gapped \\
\hline & & $A_{0}^{\prime}$ & $\widetilde{\mathrm{V}}^{\dagger} \operatorname{Diag}[1,1,1,0] \mathrm{V}$ & $U_{1}^{(1)} \times U_{3}^{(1,2)} \times U_{1}^{(2)}$ & 21 & gapless \\
\hline & & $A_{1}^{\prime}$ & $\widetilde{\mathrm{V}}^{\dagger} \operatorname{Diag}[1,1,0,0] \mathrm{V}$ & $U_{2}^{(1)} \times U_{2}^{(1,2)} \times U_{2}^{(2)}$ & 20 & gapless \\
\hline & & $A_{2}^{\prime}$ & $\widetilde{\mathrm{V}}^{\dagger} \operatorname{Diag}[1,0,0,0] \mathrm{V}$ & $U_{3}^{(1)} \times U_{1}^{(1,2)} \times U_{3}^{(2)}$ & 13 & gapless \\
\hline \multirow[t]{2}{*}{$\begin{array}{l}n_{1 e}=n_{2 h} \\
\epsilon_{Z}>0\end{array}$} & \multirow[t]{2}{*}{$U_{2}^{(1 \uparrow)} \times U_{2}^{(1 \downarrow)} \times U_{2}^{(2 \uparrow)} \times U_{2}^{(2 \downarrow)}$} & \multirow[t]{2}{*}{$B^{\prime}$} & $\left(\begin{array}{cc}0 & \mathrm{v} \\
\tilde{\mathbf{v}} & 0\end{array}\right)$ & \multirow[t]{2}{*}{$U_{2}^{(1 \uparrow, 2 \downarrow)} \times U_{2}^{(1 \downarrow, 2 \uparrow)}$} & \multirow[t]{2}{*}{8} & \multirow[t]{2}{*}{ gapped } \\
\hline & & & $\mathrm{v}, \tilde{\mathrm{v}} \in U_{2}$ & & & \\
\hline \multirow[t]{2}{*}{$\begin{array}{l}n_{1 e}>n_{2 h}{ }^{\mathrm{a}} \\
\epsilon_{Z}>0\end{array}$} & $U_{2}^{(1 \uparrow)} \times U_{2}^{(1 \downarrow)} \times U_{2}^{(2 \uparrow)} \times U_{2}^{(2 \downarrow)}$ & $A_{1}$ & $\left(\begin{array}{ll}\mathrm{v} & 0 \\
0 & 0\end{array}\right)$ & \multirow[t]{2}{*}{$U_{2}^{(1 \downarrow)} \times U_{2}^{(1 \uparrow, 2 \uparrow)} \times U_{2}^{(2 \downarrow)}$} & \multirow[t]{2}{*}{4} & \multirow[t]{2}{*}{ gapless } \\
\hline & & & $\mathrm{v} \in U_{2}$ & & & \\
\hline
\end{tabular}

$\overline{\bar{a}}$ Here, we assume parameters to be tuned so that a Fermi surface of electrons only with spin up in layer 1 coincides with the Fermi surface of holes with spin down in layer 2, for details see Fig. 6.

substituting $\mathrm{D}=\mathbb{1}$ into Eq. (17), we obtain the following structure for the order parameter in spin $\otimes$ valley space:

$$
\Delta=g_{s}(\mathbf{p}) \mathrm{V}, \quad \mathrm{V} \in U_{4} .
$$

Such a structure of the order parameter determines the symmetry group $H$ of the ground state and the degeneracy space of the order parameter, and consequently it determines the phase of the excitonic insulator.

The symmetry group $H$ of the ground state in the considered phase can be found as a group of all unitary transformations $\mathrm{U}_{H}^{(1)}, \mathrm{U}_{H}^{(2)}$ in layers 1 and 2, which leave the order parameter invariant, Eq. (10). Solving the condition (10) with the order parameter (18), we obtain matrices $\mathrm{U}_{H}^{(1)}$ and $\mathrm{U}_{H}^{(2)}$ of symmetry transformations in the layers 1 and 2, correspondingly. Thus, having $\mathrm{U}_{H}^{(1)}$ and $\mathrm{U}_{H}^{(2)}$,

$$
\mathrm{U}_{H}^{(1)}=\mathrm{U}, \quad \mathrm{U}_{H}^{(2)}=\mathrm{V}^{\dagger} \mathrm{UV}
$$

we can express an arbitrary element of the group $H$, Eq. (11), which transforms electron operators in layers 1 and 2 according to Eq. (8). Omitting indices $\zeta, \mathbf{p}, s$ we have

$$
\left(\begin{array}{l}
a_{1} \\
a_{2}
\end{array}\right) \rightarrow\left(\begin{array}{cc}
\mathrm{U} & 0 \\
0 & \mathrm{~V}^{\dagger} \mathrm{UV}
\end{array}\right)\left(\begin{array}{l}
a_{1} \\
a_{2}
\end{array}\right)
$$

Here, the matrix $\mathrm{V}$ is given by the fixed matrix of the order parameter (18). The unitary matrix $\mathrm{U} \in U_{4}$ is present in transformations in both layers. This means that the symmetry group $H$ of the ground state in the considered phase (18) consists of the combined transformations in both layers 1 and 2 . The unitary group of the combined transformations in layers
1 and 2 is denoted as $U_{4}^{(1,2)}$,

$$
\left(\begin{array}{cc}
\mathrm{U} & 0 \\
0 & \mathrm{~V}^{\dagger} \mathrm{UV}
\end{array}\right) \in U_{4}^{(1,2)} \equiv H
$$

The combined transformations from the group $U_{4}^{(1,2)}$ can also be described in terms of generators of these transformations. For this, each element of the group is written as the exponential function of the element of the group's algebra:

$$
\left(\begin{array}{cc}
\mathrm{U} & 0 \\
0 & \mathrm{~V}^{\dagger} \mathrm{UV}
\end{array}\right)=\exp \left[i \vec{\theta} \vec{\Gamma}_{H}\right] \in H
$$

where $\vec{\theta}$ is a vector of real variables and a vector $\vec{\Gamma}_{H}$ consists of generators of the group $H$. For the considered phase, these generators are

$$
\left(\Gamma_{H}\right)_{m} \equiv\left(\begin{array}{cc}
\lambda_{m} & 0 \\
0 & \mathrm{~V}^{\dagger} \lambda_{m} \mathrm{~V}
\end{array}\right), \quad m=0,1, \ldots, 15 .
$$

In contrast to Eq. (23) transformations, which change the order parameter and create a degeneracy space $G / H$, are described by the following generators:

$$
\left(\Gamma_{G / H}\right)_{m} \equiv\left(\begin{array}{cc}
\lambda_{m} & 0 \\
0 & -\mathrm{V}^{\dagger} \lambda_{m} \mathrm{~V}
\end{array}\right), \quad m=0,1, \ldots, 15
$$

Here, the $2 \times 2$ block matrices $\left(\Gamma_{H}\right)_{m}$ and $\left(\Gamma_{G / H}\right)_{m}$ act in the space of layers 1 and 2 , the matrix $\lambda_{m}$ acts on the spin $\otimes$ valley basis in the layer 1 and matrices $\pm \mathrm{V}^{\dagger} \lambda_{m} \mathrm{~V}$ acts on the basis $\Phi$ in layer 2 . The spin $\otimes$ valley basis $\Phi$ is the same in both layers. Matrices $\lambda_{m}$ are $4 \times 4$ Hermitian traceless matrices of generators of transformations from the unitary group $U_{4}$. 


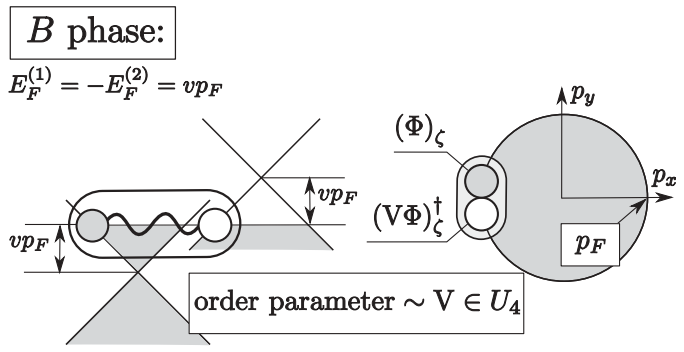

FIG. 4. In the $B$ phase within the excitonic paired state, the electron on the Fermi surface in the layer 1 (grey circle) is characterized by the index $\zeta$ in the $\operatorname{spin} \otimes$ valley basis $\Phi$, and the absent electron (white circle) in the layer 2 is characterized by the same index $\zeta$ but in the spin $\otimes$ valley basis $\mathrm{V} \Phi$, which is transformed by the matrix of the order parameter $\mathrm{V}$.

The total number of generators $\Gamma_{G / H}$, Eq. (24), equals to the dimension of the degeneracy space $G / H, \operatorname{dim}[G / H]$. In the $B$ phase, $\operatorname{dim}[G / H]=\operatorname{dim}[G]-\operatorname{dim}[H]=32-16=16$.

The electron operators in the second layer can be transformed by the matrix of the order parameter: $\mathrm{V} a_{2} \rightarrow a_{2}^{\prime}$, see Eqs. (18) and (4), or, equivalently, the spin $\otimes$ valley basis in layer 2 can be transformed by the matrix of the order parameter. In such a case from Eqs. (20) and (23) it follows, that the transformation from the group $H$, in contrast to the transformation from $G / H$, can be represented by identical transformations in both layers. These identical transformations act by the same matrix $U$ on the $\operatorname{spin} \otimes$ valley basis $\Phi$ in the layer 1 and on the transformed $\operatorname{spin} \otimes$ valley basis $\mathrm{V} \Phi$ in the layer 2, Fig. 4. Hence the matrix of the order parameter (18) defines the relative unitary rotation of the $\operatorname{spin} \otimes$ valley basis $\Phi$ in layer 2 with respect to layer 1 . It signifies the relative symmetry breaking: the ground state is not invariant under unitary transformations of the basis $\Phi$ in one layer relatively to the basis $\Phi$ in another layer. The basis $\Phi$ in layer 1 is "locked" relatively to the basis $\Phi$ in layer 2 by the matrix of the order parameter that defines the relative unitary rotation of one basis with respect to another.

Because of the presence of the relative symmetry breaking by the order parameter (18), the phase discussed here resembles the superfluid $B$ phase in the liquid Helium-3. ${ }^{38-41,46}$ In $B$ phase, the matrix of the order parameter is not degenerate. It means that all species of charge carriers develop excitonic correlations, therefore a single-particle excitation spectrum is gapped.

The external conditions $\left(\epsilon_{Z}=0, n_{1 e}=n_{2 h}\right)$ for the $B$ phase can be violated by an in-plane magnetic field or by external gates. However, the excitonic correlations continue to exist in the $B$ phase until the difference of radiuses of Fermi circles is bigger than $2 g_{+}\left(p_{F}\right) / v$, where $g_{+}\left(p_{F}\right)$ is a gap in a single-particle excitation spectrum in the $B$ phase. Indeed, such behavior can be seen, if one creates an asymmetry between charge carriers densities in layers, which can be expressed in terms of a shift $\delta E_{F}>0$ of Fermi energies: $E_{F}^{(1)}=E_{F}+\delta E_{F}, E_{F}^{(2)}=-E_{F}+\delta E_{F}$. Substituting these values to the mean-field Hamiltonian (4) and finding its eigenvalues, one obtains ${ }^{46}$ two branches of excitation spectrum $\varepsilon_{s}^{( \pm)}(p)=\sqrt{\left(s v p-E_{F}\right)^{2}+g_{s}^{2}(p)} \pm \delta E_{F}$. At values $\delta E_{F}=$ $g_{+}\left(p_{F}\right)$, one of branches of excitation spectrum becomes zero at $s=+, p=p_{F}$. At this situation the excitonic pairing stops being energetically favorable and the system appears in the normal state via a first-order phase transition. In similar way, when Fermi circles for charge carriers with opposite spin projection are separated by $2 g_{+}\left(p_{F}\right) / v$ due to an in-plane magnetic field, excitonic correlations between charge carriers on these Fermi circles vanish. This fact is schematically shown in the phase diagram Fig. 1(b): at the borders of the $B$ phase in the phase diagram excitonic correlations are no longer energetically stable and the excitonic insulator state transforms into either a normal state or into another phase via a first-order phase transition.

\section{B. The $A_{0}^{\prime}, A_{1}^{\prime}$, and $A_{2}^{\prime}$ phases}

In this section, we consider phases under the same external conditions as in $B$ phase, thus the symmetry group $G$ is again given by Eq. (7). We consider phases where order parameters are characterized by the degenerate matrices of rank $r<4$. In such phases, only a part of electron species develop excitonic correlations, therefore the single-particle excitation spectrum is gapless for certain species of electrons. The matrix of the order parameter can be chosen as follows, compare with Eq. (17):

$$
\Delta=g_{s}(\mathbf{p}) \tilde{\mathrm{V}}^{\dagger} \operatorname{Diag}[a, b, c, 0] \mathrm{V}, \quad \tilde{\mathrm{V}}^{\dagger}, \mathrm{V} \in U_{4} .
$$

Here, the diagonal matrix Diag determines the order parameter in phases, which are denoted as $A_{0}^{\prime}, A_{1}^{\prime}, A_{2}^{\prime}$ : numbers $(a, b, c)$ are given by $(1,1,1)$ in $A_{0}^{\prime}$ phase, $(1,1,0)$ in $A_{1}^{\prime}$ phase, and $(1,0,0)$ in $A_{2}^{\prime}$ phase. Using the transformed electron operators $\widetilde{\mathrm{V}} a_{1}$ in the layer 1 and $\mathrm{V} a_{2}$ in the layer 2, see Eqs. (4) and (25), the self-consistency equation on the order parameter becomes diagonal, and only first $r$ out of four equations for diagonal elements will have nonzero solutions. We assume that in the self-consistency equations we can use the screened interaction among charge carriers in the system in normal state. ${ }^{27}$ In this case, self-consistency equations in $A_{0}^{\prime}, A_{1}^{\prime}, A_{2}^{\prime}$ phases are identical to self-consistency equations in $B$ phase, therefore their nonzero solutions are given by the same gap function $g_{s}(\mathbf{p})$.

Substituting the order parameter in the symmetry condition (10) one obtains matrices of symmetry transformations in layer 1 and 2, for example for $A_{1}^{\prime}$ phase one gets

$$
\mathrm{U}_{H}^{(1)}=\widetilde{\mathrm{V}}^{\dagger}\left(\begin{array}{cc}
\mathrm{u} & 0 \\
0 & \mathrm{u}^{\prime}
\end{array}\right) \tilde{\mathrm{V}}, \quad \mathrm{U}_{H}^{(2)}=\mathrm{V}^{\dagger}\left(\begin{array}{cc}
\mathrm{u} & 0 \\
0 & \mathrm{u}^{\prime \prime}
\end{array}\right) \mathrm{V},
$$

where

$$
\mathrm{u} \in U_{2}^{(1,2)}, \quad \mathrm{u}^{\prime} \in U_{2}^{(1)}, \quad \mathrm{u}^{\prime \prime} \in U_{2}^{(2)} .
$$

Here, similarly to the $B$ phase, the $2 \times 2$ unitary matrix $u$ determines the combined unitary rotations of the first two components of the spin $\otimes$ valley basis $\widetilde{\mathrm{V}} \Phi$ in layer 1 and the first two components of the spin $\otimes$ valley basis $\mathrm{V} \Phi$ in layer 2 . Therefore such phase is characterized by a partial relative symmetry breaking. Remaining matrices $\mathrm{u}^{\prime}, \mathrm{u}^{\prime \prime} \in U_{2}$ determine independent unitary rotations of the other two components of corresponding $\operatorname{spin} \otimes$ valley basis in layers. These other two components correspond to quasiparticle's states that do not contribute to the excitonic condensation, their single-particle excitation spectrum is gappless. Therefore only two out of four 
electron's species are involved in the excitonic condensation. Because of this, such a phase is similar to the superfluid $A_{1}$ phase of liquid Helium-3, where paired states with only one spin projection $S_{z}=+1$ are present in the condensate. ${ }^{46}$ The $A_{1}$ phase of Helium-3 exists only in magnetic field, in order to underline the stability of the phase in the absence of the magnetic field we denote the phase discussed here by an additional prime, therefore it is denoted as the $A_{1}^{\prime}$ phase of the excitonic insulator. Other phases with degenerate matrices of order parameters are denoted as $A_{0}^{\prime}$ and $A_{2}^{\prime}$.

In phases $A_{0}^{\prime}, A_{1}^{\prime}$, and $A_{2}^{\prime}$ the number of nonzero diagonal elements in the diagonal matrix Diag determines the rank $r$ of the symmetry group of combined unitary rotations, denoted as $U_{r}^{(1,2)}$. Zeros in the diagonal of the matrix Diag correspond to electron states that do not develop excitonic correlations, and therefore these states can be unitary transformed independently in each layer.

Consequently, the symmetry group $H$ for $A_{0}^{\prime}, A_{1}^{\prime}$, and $A_{2}^{\prime}$ phases can be easily identified. For example, the symmetry group $H$ for $A_{1}^{\prime}$ phase is the following:

$$
H=U_{2}^{(1)} \times U_{2}^{(1,2)} \times U_{2}^{(2)} .
$$

The dimension of the degeneracy space is calculated as follows: for $A_{0}^{\prime}$ phase, $\operatorname{dim}[G / H]=32-1-9-1=21$; for $A_{1}^{\prime}$ phase, $\operatorname{dim}[G / H]=32-3 \times 4=20$; for $A_{2}^{\prime}$ phase, $\operatorname{dim}[G / H]=32-9-1-9=13$.

\section{The $B^{\prime}$ phase}

In this section, we consider the two-layer graphene system in an in-plane magnetic field. Our analysis is based on the comprehensive study by Aleiner and co-authors ${ }^{19}$ of the spontaneous symmetry breaking in graphene subjected to an in-plane magnetic field. When an in-plane magnetic field is applied, the Fermi circles for quasiparticles with different spin projections become separated due to a Zeeman splitting. Such splitting changes the symmetry group $G$, Eq. (7), of the initial Hamiltonian $\hat{H}_{\text {2layer }}$ toward a direct product of four unitary groups $U_{2}$,

$$
G=U_{2}^{(1 \uparrow)} \times U_{2}^{(1 \downarrow)} \times U_{2}^{(2 \uparrow)} \times U_{2}^{(2 \downarrow)} .
$$

Each of these $U_{2}$ groups transforms a valley space of electrons with corresponding spin projections in one layer, e.g., $U_{2}^{(1 \uparrow)}$ transforms electrons with spin up in layer 1 .

The $B^{\prime}$ phase can be obtained from the $B$ phase by the application of an in-plane magnetic field. Such magnetic field should be big enough to break the excitonic correlations in the $B$ phase and to split Fermi circles. Therefore a Zeeman energy $\epsilon_{Z}$ should be bigger than a gap in the excitation spectrum in the $B$ phase, $\epsilon_{Z}>g_{+}\left(p_{F}\right)$. In such a case, due to the initial equality of charge carrier densities $n_{1 e}=n_{2 h}$ in the $B$ phase, the two Fermi circles in layer 1 coincide with two Fermi circles in layer 2. Thus it leads to the appearance of two different Fermi circles in the system, Fig. 5. Consequently, the electron-hole pairs, which appear on different Fermi circles, have different properties: thus such electron-hole pairs have different spin projection, +1 or -1 , Fig. 5. Also due to slightly different Fermi momenta, electron-hole pairs on different Fermi circles are characterized by slightly different gap functions. Hence in

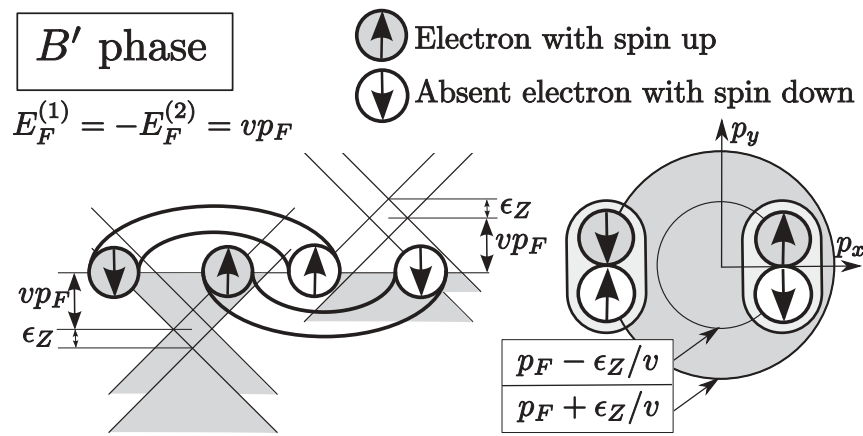

FIG. 5. Excitonic correlations in the two-layer graphene system with an in-plane magnetic field $h$ in the case of equal charge densities in layers $n_{1 e}=n_{2 h}$. Because of a Zeeman splitting $2 \epsilon_{Z}$ there are two Fermi circles with radiuses $p_{F} \pm \epsilon_{Z} / v$. The absent electron with a particular spin projection is considered as a quasiparticle (hole) with an opposite spin projection.

the $\operatorname{spin} \otimes$ valley basis $\Phi$,

$$
\Phi=\left(\uparrow \mathbf{K}_{+}, \uparrow \mathbf{K}_{-}, \downarrow \mathbf{K}_{+}, \downarrow \mathbf{K}_{-}\right),
$$

the order parameter has the following structure [compare with Eq. (18)]:

$$
\Delta=\left(\begin{array}{cc}
0 & g_{s}^{\prime}(\mathbf{p}) \mathrm{v} \\
g_{s}^{\prime \prime}(\mathbf{p}) \widetilde{\mathrm{v}} & 0
\end{array}\right) .
$$

Here matrices $\mathrm{v}$ and $\widetilde{\mathrm{v}}$ are unitary $2 \times 2$ matrices, which, by analogy with the $B$ phase, determine the relative unitary rotation of a valley space of electron states with one spin projection in layer 2 relatively to electron states with another spin projection in layer 1 . Functions $g_{s}^{\prime}(\mathbf{p}), g_{s}^{\prime \prime}(\mathbf{p})$ are gap functions, which differ from each other only because of the presence of a Zeeman splitting. However, when the Fermi energy is much bigger than Zeeman energy, $E_{F} \gg \epsilon_{Z}$, the difference between these functions is negligible and they are approximately equal to the gap function in the $B$ phase, $g_{s}^{\prime}(\mathbf{p}) \approx g_{s}^{\prime \prime}(\mathbf{p}) \approx g_{s}(\mathbf{p})$. Thus

$$
\Delta \approx g_{s}(\mathbf{p})\left(\begin{array}{ll}
0 & \mathrm{v} \\
\widetilde{\mathrm{v}} & 0
\end{array}\right) .
$$

Using this approximation, the symmetry group $H$ of the order parameter can be found from the condition (10). As a result, one obtains matrices $\mathrm{U}_{H}^{(1)}$ and $\mathrm{U}_{H}^{(2)}$ of the transformations (11) from the group $H$ in the layer 1 and 2, respectively (matrices are written in the basis (29) in each layer):

$$
\mathrm{U}_{H}^{(1)}=\left(\begin{array}{cc}
\mathrm{u} & 0 \\
0 & \widetilde{\mathrm{u}}
\end{array}\right), \quad \mathrm{U}_{H}^{(2)}=\left(\begin{array}{cc}
\widetilde{\mathrm{v}}^{\dagger} \widetilde{\mathrm{uv}} & 0 \\
0 & \mathrm{v}^{\dagger} \mathrm{uv}
\end{array}\right) .
$$

The corresponding electron operators are transformed as follows:

$$
\begin{aligned}
& a_{1, \uparrow} \rightarrow \mathrm{u} a_{1, \uparrow}, \quad a_{2, \downarrow} \rightarrow \mathrm{v}^{\dagger} \mathrm{uv} a_{2, \downarrow}, \quad \mathrm{u} \in U_{2}^{(1 \uparrow, 2 \downarrow)}, \\
& a_{1, \downarrow} \rightarrow \widetilde{\mathrm{u}} a_{1, \downarrow}, \quad a_{2, \uparrow} \rightarrow \widetilde{\mathrm{v}}^{\dagger} \widetilde{\mathrm{u}} \widetilde{v} a_{2, \uparrow}, \quad \widetilde{\mathrm{u}} \in U_{2}^{(1 \downarrow, 2 \uparrow)} .
\end{aligned}
$$

The unitary $2 \times 2$ matrix u, Eq. (32), determines a subgroup of the group $H$, which consists of combined unitary rotation of valley space of electrons with spin up in layer 1 and electrons 
with spin down in layer $2: \mathrm{u} \in U_{2}^{(1 \uparrow, 2 \downarrow)}$. The unitary $2 \times 2$ matrix $\widetilde{u}$, Eq. (33), defines another corresponding subgroup of the group $H, \tilde{\mathrm{u}} \in U_{2}^{(1 \downarrow, 2 \uparrow)} \subset H$. Hence in the phase considered here, the symmetry group $H$ of the order parameter is given by direct product of two subgroups:

$$
H=U_{2}^{(1 \uparrow, 2 \downarrow)} \times U_{2}^{(1 \downarrow, 2 \uparrow)} .
$$

Using expressions for groups $G$ and $H$ in the $B^{\prime}$ phase, we found that the degeneracy space $G / H$ is eight dimensional, $\operatorname{dim}[G / H]=4 \times 4-4-4=8$. It is also defined by the structure of the order parameter (30), i.e., here, the degeneracy space is determined as a space of all possible unitary $2 \times 2$ matrices $v$ and $\widetilde{v}$. Because of the nondegenerate matrix of the order parameter, the single-particle excitation spectrum in this phase is gapped.

Similarly to the $B$ phase, the excitonic correlations in the $B^{\prime}$ phase cease to exist when the external conditions $\left(\epsilon_{Z}>\right.$ $\left.g_{+}\left(p_{F}\right), n_{1 e}=n_{2 h}\right)$ are perturbed, i.e., when Fermi circles in different layers are separated for the energy interval which is bigger than a double value of a gap in the single-particle excitation spectrum. Thus, in particular, in the schematic phase diagram Fig. 1(b) at the border of the $B^{\prime}$ phase (when a symmetry $n_{1 e}=n_{2 h}$ of charge carriers densities is violated) the ground state of the system transforms to an uncorrelated normal ground state via the first-order phase transition.

\section{The $A_{1}$ phase}

In contrast to $B$ and $B^{\prime}$ phases, where all species of charge carriers develop excitonic correlations, in this section, we discuss another possible realization of the excitonic insulator state in the two-layer graphene system. We show that the excitonic correlated state can exist in the presence of an in-plane magnetic field and a specially chosen asymmetry in charge carriers densities in layers.

In order to achieve a necessary external conditions, firstly, we consider the two-layer system without a magnetic field and with equal charge carrier densities in layers. Under such conditions, the spectrum of electrons in both layers have only one Fermi circle at the Fermi momentum $p_{F}$. By changing the external gate voltages, we create asymmetry between charge carrier densities in layers: $n_{1 e}>n_{2 h}$. Thus the Fermi circle in layer 1 is situated at the momentum $p_{F}+\delta p_{F}$, and the Fermi circle in layer 2 is situated at the momentum $p_{F}-\delta p_{F}$, where $\delta p_{F}>0$, and $p_{F}$ is the Fermi momentum in the case $n_{1 e}=n_{2 h}$ (i.e., in $B$ and $B^{\prime}$ phases). It is assumed that the separation between Fermi circles is big enough to prevent the development of excitonic correlations. Keeping the chosen values of densities, we switch-on an in-plane magnetic field with such a magnitude that the Zeeman energy $\epsilon_{Z}$ is equal to the energy shift of each Fermi surface, $\epsilon_{Z}=v \delta p_{F}$, Fig. 6 . The presence of an in-plane magnetic field signifies that the symmetry group $G$ in such case is the same as in $B^{\prime}$ phase.

The external conditions mentioned above lead to the situation when only two out of four Fermi circles coincide: both the Fermi circle of electrons with spin up in the layer 1 (a Fermi circle with a smaller radius in the layer 1) and the Fermi circle of electrons with spin up in the layer 2 (a Fermi circle with a bigger radius in the layer 2) are situated at the same Fermi momenta $p_{F}$. Thus electron-hole pairs are formed

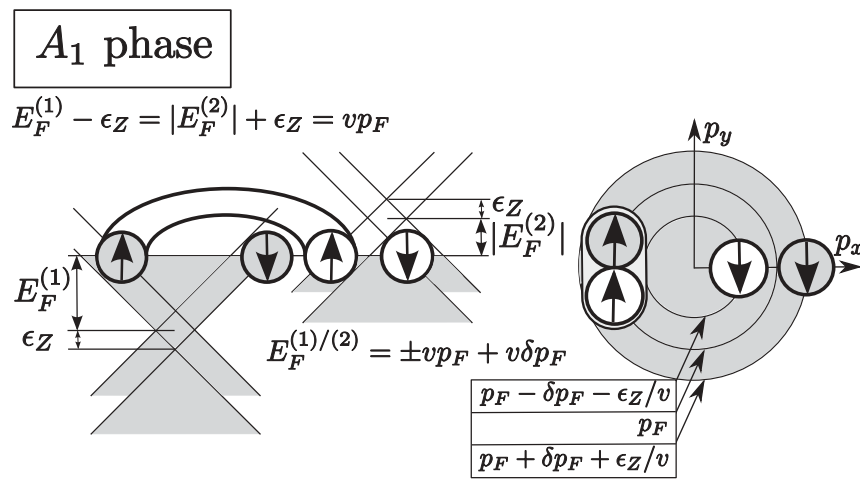

FIG. 6. Excitonic correlations in the $A_{1}$ phase. Starting from a zero magnetic field the asymmetry between charge carrier densities $n_{1 e}>n_{2 h}$ is created. In terms of Fermi energies, it means $E_{F}^{(1)}>$ $\left|E_{F}^{(2)}\right|$. A magnitude of an in-plane magnetic field is chosen such that a Zeeman energy $\epsilon_{Z}$ satisfies the condition: $E_{F}^{(1)}-\epsilon_{Z}=\left|E_{F}^{(2)}\right|+\epsilon_{Z}$, where $\left(E_{F}^{(1)}-\epsilon_{Z}\right) / v$ is the radius of the Fermi circle for electrons with spin up in layer 1 , and $\left(\left|E_{F}^{(2)}\right|+\epsilon_{Z}\right) / v$ is a radius of the Fermi circle for electrons with spin up in layer 2. Both of these Fermi circles are situated at the same Fermi momentum $p_{F}$. Therefore two out of four Fermi circles coincide, leading to excitonic correlations between only half of electron's species. Using the expression for the Fermi energies $E_{F}^{(1) /(2)}= \pm v p_{F}+v \delta p_{F}, p_{F}>\delta p_{F}$, where $\delta p_{F}=$ $\left(\sqrt{n_{1 e}}-\sqrt{n_{2 h}}\right) \sqrt{\pi} / 2$, the condition on the Zeeman energy is the following: $\epsilon_{Z}=v \delta p_{F}$.

on these two coincided Fermi circles. Notice that a total spin projection of such an electron-hole pair is equal to a zero in contrast to electron-hole pairs with spin projections +1 or -1 in the $B^{\prime}$ phase. Fermi circles for electrons with spin down in both layers do not coincide with any other Fermi surfaces. Therefore corresponding electrons and holes are in a normal state (i.e., they do not participate in excitonic correlations), their single-particle excitation spectrum is gapless.

Thus for the phase discussed here only half of all electron species in the system develop excitonic correlations. It is reflected in the order parameter, whose structure in the basis (29) in both layers is given by the following expression:

$$
\Delta=g_{s}(\mathbf{p})\left(\begin{array}{ll}
\mathrm{v} & 0 \\
0 & 0
\end{array}\right) .
$$

Here, the gap function $g_{s}(\mathbf{p})$ is the same as in other phases due to the same Fermi momentum $p_{F}$ in the self-consistency equation and due to the approximation of the interaction among charge carriers in all phases by the screened interaction among charge carriers in the system in normal state. Similarly to the phases discussed previously, the unitary $2 \times 2$ matrix $\mathrm{v}$ in the order parameter (35) determines a relative unitary rotation of the valley space of electrons with spin up in layer 2 relatively electrons with spin up in layer 1.

Solving the condition (10) with the order parameter (35), we found that transformations from the group $H$ are represented in layer 1 and 2 by following matrices $\mathrm{U}_{H}^{(1)}$ and $\mathrm{U}_{H}^{(2)}$ respectively (both matrices are written in the basis (29) in each layer):

$$
\mathrm{U}_{H}^{(1)}=\left(\begin{array}{cc}
\mathrm{u} & 0 \\
0 & \mathrm{u}^{\prime}
\end{array}\right), \quad \mathrm{U}_{H}^{(2)}=\left(\begin{array}{cc}
\mathrm{v}^{\dagger} \mathrm{uv} & 0 \\
0 & \mathrm{u}^{\prime \prime}
\end{array}\right) .
$$


Here matrices $\mathrm{u}, \mathrm{u}^{\prime}, \mathrm{u}^{\prime \prime}$ are unitary $2 \times 2$ matrices. The matrix $\mathrm{u}$ performs a combined unitary transformation of a valley space of electrons with spin up in both layers, in addition, the valley space of electrons in layer 2 is rotated by the order parameter (35), compare with Eq. (32). The valley space of electrons with spin down is transformed by the unitary matrix $\mathrm{u}^{\prime}$ in layer 1 and by the unitary matrix $\mathrm{u}^{\prime \prime}$ in layer 2 , correspondingly:

$$
\begin{gathered}
a_{1, \uparrow} \rightarrow \mathrm{u} a_{1, \uparrow}, \quad a_{2, \uparrow} \rightarrow \mathrm{v}^{\dagger} \mathrm{uv} a_{2, \uparrow}, \quad \mathrm{u} \in U_{2}^{(1 \uparrow, 2 \uparrow)}, \\
a_{1, \downarrow} \rightarrow \mathrm{u}^{\prime} a_{1, \downarrow}, \quad \mathrm{u}^{\prime} \in U_{2}^{(1 \downarrow)}, \\
a_{2, \downarrow} \rightarrow \mathrm{u}^{\prime \prime} a_{2, \downarrow}, \quad \mathrm{u}^{\prime \prime} \in U_{2}^{(2 \downarrow)} .
\end{gathered}
$$

Thus group $\mathrm{H}$ consists of the direct product of three unitary groups:

$$
H=U_{2}^{(1 \downarrow)} \times U_{2}^{(1 \uparrow, 2 \uparrow)} \times U_{2}^{(2 \downarrow)} .
$$

Using the expressions for initial symmetry group $G$ and Eq. (40), we find that the degeneracy space $G / H$ in this phase is four dimensional, $\operatorname{dim}[G / H]=16-3 \times 4=4$. It is determined by the manifold of all possible matrices $\mathrm{v} \in U_{2}$ in the structure of the order parameter (35).

The subgroups $U_{2}^{(1 \downarrow)}$ and $U_{2}^{(2 \downarrow)}$ are present in both groups $G$ and $H$, the appearance of the correlated state does not change them. Therefore for the phase discussed here the initial symmetry is broken only partially. According to the similarities with the superfluid $A_{1}$ phase of liquid Helium-3 (i.e., that the phase described here exists in magnetic field and has a partial relative symmetry breaking ${ }^{38-41,46}$ ), the phase discussed in this subsection was denoted as $A_{1}$ phase.

\section{RESULTS AND DISCUSSIONS}

In the present paper, we consider a two-layer graphene system where external gate voltage induces a finite density of electrons in one layer and holes in another. Assuming that the transition temperature $T_{c}$ toward excitonic insulator is high enough so that it can be observed, we classify phases of such correlated state. In order to obtain different excitonic correlations and therefore different phases, we propose to use parallel to graphene layers magnetic field and perpendicular to graphene layers electric field.

Firstly, we consider the Hamiltonian of the two-layer graphene system. We recognize that the ground state is characterized by a high symmetry group - the group of unitary rotations of spin $\otimes$ valley space of electrons in each layer independently. Below a transition temperature $T_{c}$, this symmetry is reduced by a nonzero order parameter toward a symmetry of the excitonic insulating ground state, which consists of electron-hole pairs with electrons on one layer and holes on another. Following the BCS theory of superconductivity, we identify the condition for such electron-hole pairing, determine the order parameter and build a BCS-like mean-field theory of the excitonic insulator. Analyzing a symmetry breaking of the initial ground state by the order parameter, we consider a condition that mutually determines the order parameter and the corresponding symmetry group of the excitonic insulator ground state. Using a singular value decomposition of the matrix of the order parameter, for each phase of the excitonic insulator, we obtain a corresponding symmetry group of the ground state, a structure of the order parameter and its degeneracy space. The results of a phase classification of the excitonic insulator are shown in Table I, the most energetically stable phases are shown in the phase diagram, Fig. 1(b), and on Figs. 4-6.

It is important to notice that the excitonic correlations in all phases discussed in this paper origin from the coincided Fermi surfaces at approximately the same Fermi momentum $p_{F}$ (we use assumption $E_{F} \gg \epsilon_{Z}$, where the Fermi energy $E_{F}$ is determined in the system without a magnetic field and with equal densities of charge carriers in layers $n_{1 e}=n_{2 h}$ ). Thus assuming that the interaction among charge carriers is the same in all phases (i.e., that the effect of excitonic correlations on the screening of the interaction can be neglected ${ }^{27}$ ), we obtain that the energy gap in the single-particle excitation spectrum in all phases is determined by the same self-consistency equation. Therefore the transition temperature $T_{c}$ estimated from the self-consistency equation ${ }^{46}$ should be the same for all phases.

At a temperature lower than the transition temperature $T_{c}$, transitions between phases in the phase diagram are found to be of the first order. Phases of excitonic insulator have different properties: thus the electron-hole pairs in $B^{\prime}$ phase have total spin projection +1 or -1 , see Fig. 5 , whereas in the $A_{1}$ phase a total spin projection of an electron-hole pair is equal to zero, see Fig. 6.

According to the number of estimations of the critical temperature in the considered system, the most optimistic estimation gives values of $T_{c}$ close to a room temperature. ${ }^{22}$ However, this estimation ${ }^{22}$ does not take screening of the Coulomb interaction into account, explaining it by the assumption of the first-order phase transition in the system. Some other estimations ${ }^{25,27}$ point on the improbability of observation of excitonic condensation due to extremely low transition temperature $\lesssim 1 \mathrm{mK},\left(T_{c} \approx 10^{-7} E_{F}\right)$. According to Refs. 25 and 27 , the reason for low transition temperature lays in the effective screening of the Coulomb interaction by a big number $N$ of species of electrons. ${ }^{25,51}$ In the considered system $N=8$, which is given by the product of two valleys, two spin projections and two layers. Such large $N$ increases screening and makes excitonic condensation not so effective, as in the monolayer graphene $\mathrm{e}^{52}$ and especially in the monolayer graphene in a magnetic field, ${ }^{19,53}$ where $T_{c}$ can reach values up to $10^{-4} E_{F}$. However, recent investigations, based on a detailed treatment of the screened Coulomb interaction ${ }^{28-30,54,55}$ and on a consideration of a multiband pairing ${ }^{31,54}$ or pairing with nonzero momentum ${ }^{56}$ suggest that the transition temperature $T_{c}$ can be sufficiently big for the experimental observation of the excitonic insulator in the considered system. Together with recent experimental realization of the two-layer graphene system $^{32-37}$ it provides a hope that the phase diagram of the excitonic insulator, Fig. 1(b), under favorable conditions ${ }^{57-60}$ will be observed experimentally.

\section{ACKNOWLEDGMENTS}

Y.F.S. would like to thank L. Glazman, E. Burovski, and Y. Sherkunov for useful discussions. The authors thank EPSRC and Physics Department at Lancaster University for financial support. 
*y.suprunenko@lancaster.ac.uk

${ }^{1}$ J. M. Blatt et al., Phys. Rev. 126, 1691 (1962).

${ }^{2}$ L. V. Keldysh and Y. V. Kopaev, Sov. Phys. Solid State 6, 2219 (1965).

${ }^{3}$ D. Jerome, T. M. Rice, and W. Kohn, Phys. Rev. 158, 462 (1967).

${ }^{4}$ L. V. Keldysh and A. N. Kozlov, Sov. Phys. JETP 27, 521 (1968).

${ }^{5}$ B. I. Halperin and T. M. Rice, Rev. Mod. Phys. 40, 755 (1968).

${ }^{6}$ Yu. E. Lozovik, V. I. Yudson, JETP Lett. 22, 274 (1975).

${ }^{7}$ S. I. Shevchenko, Fiz. Nizk. Temp. 2, 505 (1976) [Sov. J. Low Temp. Phys. 2, 251 (1976)].

${ }^{8}$ U. Sivan, P. M. Solomon, and H. Shtrikman, Phys. Rev. Lett. 68, 1196 (1992)

${ }^{9}$ L. V. Butov, A. Zrenner, G. Abstreiter, G. Bohm, and G. Weimann, Phys. Rev. Lett. 73, 304 (1994).

${ }^{10}$ K. Moon, H. Mori, K. Yang, S. M. Girvin, A. H. MacDonald, L. Zheng, D. Yoshioka, and S.-C. Zhang, Phys. Rev. B 51, 5138 (1995).

${ }^{11}$ X. Zhu, P. B. Littlewood, M. S. Hybertsen, and T. M. Rice, Phys. Rev. Lett. 74, 1633 (1995).

${ }^{12}$ I. B. Spielman, J. P. Eisenstein, L. N. Pfeiffer, and K. W. West, Phys. Rev. Lett. 84, 5808 (2000); 87, 036803 (2001).

${ }^{13}$ J. P. Eisenstein and A. H. MacDonald, Nature (London) 432, 691 (2004).

${ }^{14}$ A. A. High, J. R. Leonard, A. T. Hammack, M. M. Fogler, L. V. Butov, A. V. Kavokin, K. L. Campman, and A. C. Gossard, Nature (London) 483, 584 (2012).

${ }^{15}$ K. S. Novoselov et al., Science 306, 666 (2004).

${ }^{16}$ K. S. Novoselov et al., Nature (London) 438, 197 (2005).

${ }^{17}$ Y. Zhang, J. P. Small, M. E. S. Amori, and P. Kim, Phys. Rev. Lett. 94, 176803 (2005).

${ }^{18}$ Y. Zhang et al., Nature (London) 438, 201 (2005).

${ }^{19}$ I. L. Aleiner, D. E. Kharzeev, and A. M. Tsvelik, Phys. Rev. B 76, 195415 (2007).

${ }^{20}$ Yu. E. Lozovik and A. A. Sokolik, JETP Lett. 87, 55 (2008).

${ }^{21}$ Yu. E. Lozovik, S. P. Merkulova, and A. A. Sokolik, Usp. Fiz. Nauk 178, 757 (2008) [Phys. Usp. 51, 727 (2008)] (in Russian).

${ }^{22}$ H. Min, R. Bistritzer, J. J. Su, and A. H. MacDonald, Phys. Rev. B 78, 121401(R) (2008).

${ }^{23}$ C.-H. Zhang and Yogesh N. Joglekar, Phys. Rev. B 77, 233405 (2008).

${ }^{24}$ Yu. E. Lozovik, A. A. Sokolik, and M. Willander, Phys. Status Solidi A 206, 927 (2009).

${ }^{25}$ M. Y. Kharitonov and K. B. Efetov, Phys. Rev. B 78, 241401(R) (2008).

${ }^{26}$ R. Bistritzer, H. Min, J.-J. Su, and A. H. MacDonald, arXiv:0810.0331 [cond-mat].

${ }^{27}$ M. Y. Kharitonov and K. B. Efetov, Semicond. Sci. Technol. 25, 034004 (2010).

${ }^{28}$ Yu. E. Lozovik and A. A. Sokolik, Phys. Lett. A 374, 326 (2009).

${ }^{29}$ Yu. E. Lozovik and A. A. Sokolik, Eur. Phys. J. B 73, 195 (2010).

${ }^{30}$ Yu. E. Lozovik, S. L. Ogarkov, and A. A. Sokolik, Philos. Trans. R. Soc. London A 368, 5417 (2010).
${ }^{31}$ M. P. Mink, H. T. C. Stoof, R. A. Duine, and A. H. MacDonald, Phys. Rev. B 84, 155409 (2011).

${ }^{32}$ H. Schmidt, T. Ludtke, P. Barthold, E. McCann, V. I. Fal'ko, and R. J. Haug, Appl. Phys. Lett. 93, 172108 (2008).

${ }^{33}$ H. Schmidt, T. Ludtke, P. Barthold, and R. J. Haug, Phys. Rev. B 81, 121403(R) (2010).

${ }^{34}$ T. Ludtke, H. Schmidt, P. Barthold, and R. J. Haug, Physica E 42, 695 (2010).

${ }^{35}$ H. Schmidt, T. Ludtke, P. Barthold, and R. J. Haug, Physica E 42, 699 (2010).

${ }^{36}$ D. Rainis, F. Taddei, M. Polini, G. Leon, F. Guinea, and V. I. Fal'ko, Phys. Rev. B 83, 165403 (2011).

${ }^{37}$ L. A. Ponomarenko, A. A. Zhukov, R. Jalil, S. V. Morozov, K. S. Novoselov, V. V. Cheianov, V. I. Falko, K. Watanabe, T. Taniguchi, A. K. Geim, and R. V. Gorbachev, Nat. Phys. 7, 958 (2011).

${ }^{38}$ A. J. Leggett, Rev. Mod. Phys. 47, 331 (1975).

${ }^{39}$ J. C. Wheatley, Rev. Mod. Phys. 47, 415 (1975).

${ }^{40}$ V. P. Mineev, Usp. Fiz. Nauk 139, 303 (1983) [Sov. Phys.- Uspekhi 26, 160 (1983)].

${ }^{41}$ G. E. Volovik, "Symmetry in Superluid 3He," in Modern Problems in Condensed Matter Sciences (Helium Three), edited by W. P. Halperin and L. P. Pitaevskii, Chap. 2 (North- Holland, 1990).

${ }^{42}$ D. Vollhardt and P. Wolfle, The Superfluid Phases of Helium 3 (Taylor and Francis, New York, 1990).

${ }^{43}$ P. R. Wallace, Phys. Rev. 71, 622 (1947).

${ }^{44}$ A. H. Castro Neto, F. Guinea, N. M. R. Peres, K. S. Novoselov, and A. K. Geim, Rev. Mod. Phys. 81, 109 (2009); e-print arXiv:0709.1163v2.

${ }^{45}$ N. M. R. Peres, J. Phys.: Condens. Matter 21, 323201 (2009).

${ }^{46}$ V. P. Mineev and K. V. Samokhin, Introduction to Unconventional Superconductivity (Gordon and Breach Science Publishers, 1999).

${ }^{47}$ M. Katsnelson, K. Novoselov, and A. Geim, Nat. Phys. 2, 620 (2006).

${ }^{48}$ J. Bardeen, L. N. Cooper, and J. R. Schrieffer, Phys. Rev. 108, 1175 (1957).

${ }^{49}$ C. Bruder and D. Vollhardt, Phys. Rev. B 34, 131 (1986).

${ }^{50}$ A. Howard, Elementary Linear Algebra: Applications Vversion, 6th ed. (Wiley, New York, 1991).

${ }^{51}$ M. S. Foster and I. L. Aleiner, Phys. Rev. B 77, 195413 (2008).

${ }^{52}$ D. V. Khveshchenko, Phys. Rev. Lett. 87, 206401 (2001); 87, 246802 (2001)

${ }^{53}$ E. V. Gorbar, V. P. Gusynin, V. A. Miransky, and I. A. Shovkovy, Phys. Rev. B 66, 045108 (2002).

${ }^{54}$ Yu. E. Lozovik, S. L. Ogarkov, and A. A. Sokolik, Phys. Rev. B 86, 045429 (2012)

${ }^{55}$ I. Sodemann, D. A. Pesin, and A. H. MacDonald, Phys. Rev. B 85, 195136 (2012).

${ }^{56}$ D. K. Efimkin and Yu. E. Lozovik, JETP 113, 880 (2011).

${ }^{57}$ R. Bistritzer and A. H. MacDonald, Phys. Rev. Lett. 101, 256406 (2008).

${ }^{58}$ D. Basu, L. F. Register, A. H. MacDonald, and S. K. Banerjee, Phys. Rev. B 84, 035449 (2011).

${ }^{59}$ D. K. Efimkin, V. A. Kulbachinskii, and Yu. E. Lozovik, JETP Lett. 93, 219 (2011)

${ }^{60}$ J.-J. Su and A. H. MacDonald, Nat. Phys. 4, 799 (2008). 